A more crucial test of Gibbs's law will be undertaken with homologue-free $\mathbf{C}_{0^{-}}$and $\mathbf{C}_{\mathbf{1 4}}$-sulphates. In addition, investigations are being made on mixtures of surface-active agents in solution, co-adsorption of ions, etc., using the radioactive method.

\section{Gunnar Aniansson}

Department of Physical Chemistry

OLE LAMM

Royal Institute of Technology,

Stockholm 26.

Oct. 20 .

"Adam, "The Physics and Chemistry of Surfaces", 113 and 409 (London, 1941).

${ }^{2}$ Lottermoser-Püschel, Koll. Z., 68, 185 (1934).

\section{Light Output from a Spark 'Point Source'}

Is view of the increasing use of high-speed shadow and schlieren photography by short-duration flashes from electric discharges, it seemed desirable to determine the various factors affecting the light output, so that desired characteristics could be obtained in the most convenient way.

It has been found that the light emitted by a given spark gap depends on the current in the gap and on its variation with time. The light is only affected by the voltage, capacitance and inductance of the disoharge circuit in so far as these affect the current. For example, the same light output is obtained by the discharge of a $0 \cdot 1 \mu F$. condenser at $10 \mathrm{kV}$. through a circuit inductance of $0.4 \mu \mathrm{H}$. as by the discharge of a $0.033 \mu \mathrm{F}$. condenser at $30 \mathrm{kV}$. through a circuit inductance of $1.2 \mu \mathrm{H}$. giving the same oscillatory current of $5 \mathrm{k}$.amp. with period of $1.3 \mu \mathrm{sec}$. The upper curves in Fig. 1 indicate the dependence on current of the relative maximum light output (as measured by a photomultiplier, amplifier and cathode ray oscillograph) from a 1-mm. gap in air between rod electrodes carrying a lightly damped oscillatory current of period 1.3 usec. The variation of light output with time is shown in Fig. 2, curve $(b)$, which indicates that a substantial proportion of the total light is emitted in the first half-cycle of the discharge.

The peak light output is reduced if the time to maximum current exceeds $1 \mu$ sec., and the lower curves in Fig. 1 give the relative maximum light output for a discharge of period $40 \mu$ sec. With such a discharge between copper electrodes, the second and

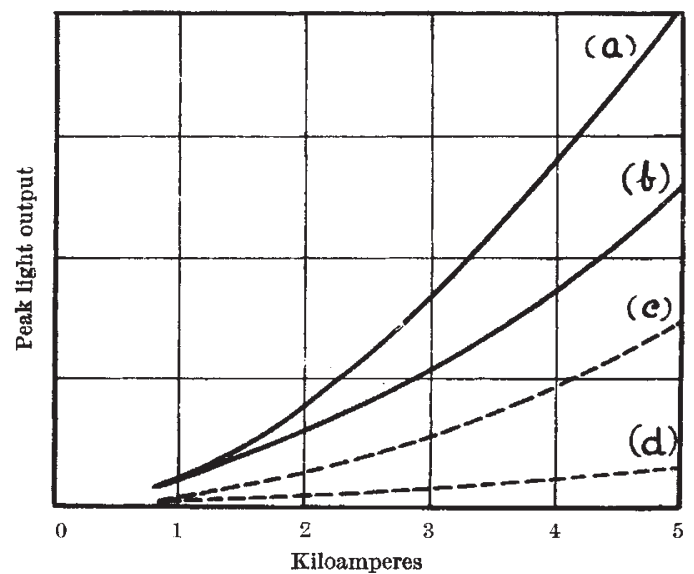

Fig. 1. Variation of peak light with current in 1-mm. gap : (a) and (c) cadmium electrodes; (b) and $(d)$ copper electrodes $(a)$ and $(b)$ discharge oscillation period $1.3 \mu$ sec.; (c) and $(d)$ discharge oscillation period $40 \mu$ sec.

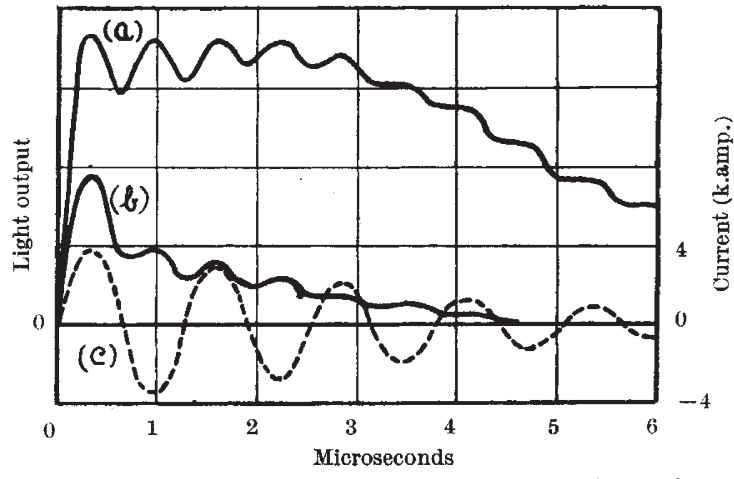

Fig. 2. Variation of current and light with time: $(a)$ light from constricted $3-\mathrm{mm}$. axial gap; $(b)$ light from open 1-mm. gap ; (c) current

third half-cycles produce as much light as the first, suggesting that a stable arc condition has been established in a few microseconds. It is believed that, with discharges of the shorter period, a higher voltage remains on the gap during the first quarter-cycle, so that more energy is dissipated in the gap. It is intended to make voltage measurements.

The maximum light output is greater for the more volatile metals, and the rate of decrease is less. For example, cadmium electrodes $1 \mathrm{~mm}$. apart give about 50 per cent higher maximum, and 100 per cent more total light than copper electrodes.

An alternative form of spark, emitting light axially through a $1 \cdot \mathrm{mm}$. hole in one electrode, has been examined. If the spark is not constricted, it is usually not axial, and less light is available than is emitted radially from a $1-\mathrm{mm}$. gap between rods. With the discharge constricted by an insulating tube, measurements have been made on sparks $1 \frac{1}{2}-8 \mathrm{~mm}$. long. The maximum light emitted was about twice that from the open 1-mm. gap. The total light, as measured by a photo-electric integrating flashmeter, increased with gap-length, varying from five to eight times that for the open gap. A photomultiplier oscillogram showed that the increase in total light was obtained at a cost of increased duration, as much light being emitted during the second discharge halfcycle as in the first (Fig. 2, curve $a$ ).

The measurements of total light, for a discharge current of $4 \mathrm{k}$.amp., period $1.3 \mu \mathrm{sec}$., indicate that the 1-mm. open gap with copper electrodes, the I-mm. open gap with cadmium or magnesium electrodes, and the constricted axial gaps with brass electrodes, were respectively equivalent to about $0 \cdot 1$, 0.2 and $0.6-0.9$ candle-second.

$$
\text { W. G. STANDRING }
$$

$$
\text { J. S. T. Looms }
$$

Electricity Division,

National Physical Laboratory. Sept. 29.

\section{Dielectric Behaviour of Methyl Palmitate in the Microwave Region}

THE variation of the dielectric constant and loss of methyl palmitate at room temperature over the frequency range $50 \mathrm{c} . / \mathrm{s}$. to $3 \times 10^{10} \mathrm{c}$. $/ \mathrm{s}$. was first reported by Dryden and Willis Jackson ${ }^{1}$. The evidence provided for resonance absorption in the frequency range centred on $10^{10} \mathrm{c} . / \mathrm{s}$. led us to investigate the absorption at room temperature and at as many 